\title{
Equações de regressão para estimar valores energéticos do grão de trigo e seus subprodutos para frangos de corte, a partir de análises químicas
}

\author{
[Regression equations to evaluate the energy values of wheat grain and its by-products \\ for broiler chickens from chemical analyses]

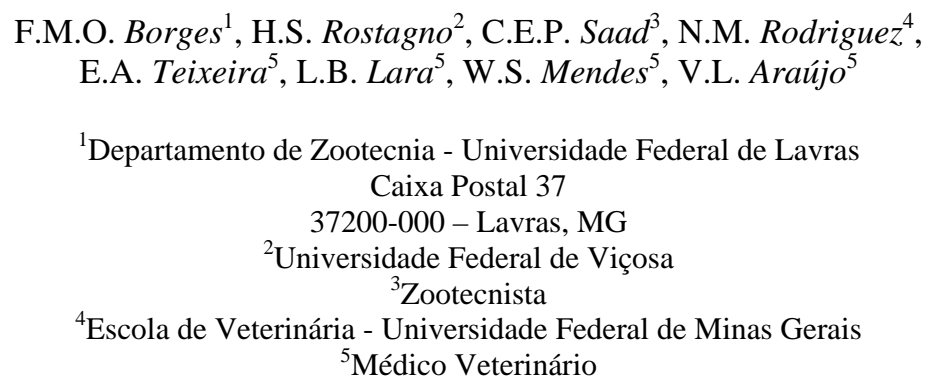

Recebido para publicação, em 13 de fevereiro de 2003

Recebido para publicação, após modificações, em 22 de setembro de 2003

E-mail: borgesvet@ufla.br

\section{RESUMO}

Um experimento foi realizado com frangos de corte para a obtenção de equações de predição da energia a partir de análises químicas e dados de energia metabolizável (EM) utilizando como alimentos-teste o trigo e alguns de seus produtos, comparando quatro metodologias de estimação. Sete alimentos oriundos do trigo e uma ração-referência foram utilizados nos cinco tratamentos: trigo integral moído, gérmen de trigo, farinha de trigo clara, farinha de trigo escura, farelo de trigo para uso humano, farelo de trigo para uso animal e farelo de trigo grosso. Com base nas análises químicas de fibra bruta (FB), extrato etéreo (EE), proteína $(\mathrm{PB})$, cinzas $(\mathrm{Cz})$ e amido $(\mathrm{A})$ dos alimentos e com os resultados observados de EM aparente (EMA), EM verdadeira (EMV), EM aparente corrigida $\left(E^{2} A_{n}\right)$ e EM verdadeira corrigida pelo balanço de nitrogênio $\left(\mathrm{EMV}_{\mathrm{n}}\right)$ nos cinco tratamentos, desenvolveram-se equações de predição por stepwise. FB foi a variável que melhor se relacionou com os valores de energia metabolizável, entretanto esse valor isolado não foi suficiente para uma boa estimativa dos valores energéticos ( $\mathrm{R}^{2}$ abaixo de 0,80$)$. Quando somada ao EE e à PB aumentou-se a precisão das equações, com valores de $\mathrm{R}^{2}$ acima de 0,90 na maioria das equações. Estimativas a partir dos valores de todos tratamentos resultaram em equações menos precisas para EMA, com menores $\mathrm{R}^{2}$. Quando se utilizaram somente dados dos tratamentos pela metodologia tradicional ou de alimentação forçada separadamente aumentou-se a precisão das equações, com $\mathrm{R}^{2}$ acima de 0,85. Para EMV e EMV , as equações lineares múltiplas, utilizando-se FB, EE e PB, apresentaram boa precisão $\left(\mathrm{R}^{2}>0,90\right)$, independente da utilização de todos os dados experimentais ou seu agrupamento por metodologia. Para a $\mathrm{EMV}_{\mathrm{n}}$, além da alta precisão, os valores do coeficiente linear (a) das equações foram muito próximos quando se utilizaram todos os tratamentos ou agrupando-os por metodologia, o que significou pouca influência da metodologia sobre essa medida. A FDN não mostrou ser melhor preditor de EM do que a FB.

Palavras-chave: frango, trigo, energia metabolizável, equação de predição

\section{ABSTRACT}

One experiment was run with broiler chickens, to obtain prediction equations for metabolizable energy (ME) based on feedstuffs chemical analyses, and determined ME of wheat grain and its by-products, 
using four different methodologies. Seven wheat grain by-products were used in five treatments: wheat grain, wheat germ, white wheat flour, dark wheat flour, wheat bran for human use, wheat bran for animal use and rough wheat bran. Based on chemical analyses of crude fiber (CF), ether extract (EE), crude protein (CP), ash (AS) and starch (ST) of the feeds and the determined values of apparent energy (MEA), true energy (MEV), apparent corrected energy (MEAn) and true energy corrected by nitrogen balance $\left(M E V_{n}\right)$ in five treatments, prediction equations were obtained using the stepwise procedure. CF showed the best relationship with metabolizable energy values, however, this variable alone was not enough for a good estimate of the energy values ( $R^{2}$ below 0.80). When EE and CP were included in the equations, $R^{2}$ increased to 0.90 or higher in most estimates. When the equations were calculated with all treatments, the equation for MEA were less precise and $R^{2}$ decreased. When $M E$ data of the traditional or force-feeding methods were used separately, the precision of the equations increases ( $R^{2}$ higher than 0.85). For MEV and $M E V_{n}$ values, the best multiple linear equations included $C F, E E$ and $C P\left(R^{2}>0.90\right)$, independently of using all experimental data or separating by methodology. The estimates of $M E V_{n}$ values showed high precision and the linear coefficients (a) of the equations were similar for all treatments or methodologies. Therefore, it explains the small influence of the different methodologies on this parameter. NDF was not a better predictor of ME than $C F$.

Keywords: bird, wheat, metabolizable energy, prediction equation

\section{INTRODUÇÃO}

Na formulação de rações normalmente utilizam-se valores bromatológicos extraídos de tabelas de composição de alimentos, entretanto, esses valores podem ser diferentes entre as várias tabelas segundo Rostagno (1990). Essas diferenças podem ser atribuídas às diferentes proporções e tipos de matéria-prima utilizada, além de diferenças no processamento desses alimentos.

Segundo Sakamura e Silva (1998), o conteúdo em nutrientes de vários cereais encontrados nas tabelas de composição não são confiáveis para a formulação de rações, e entre os fatores que determinam essa diversidade pode-se citar a variedade. Vohra (1980), citado por Sakamura e Silva (1998), observou diferenças nos valores de energia metabolizável de 3180 a $3920 \mathrm{kcal} / \mathrm{kg}$ de MS entre variedades de sorgo.

Dessa forma, torna-se inseguro para a indústria utilizar os valores de tabela e seria extremamente oneroso e difícil submeter todas as partidas de matéria-prima a ensaios in vivo. Uma vez que essas mesmas indústrias podem obter, com relativa facilidade, determinações químicas como os teores de proteína, fibra, extrato etéreo etc, o uso de regressões baseadas nessas análises poderia ser de grande valia.

Segundo Albino (1980), a importância em determinar equações de predição para o valor energético dos alimentos baseia-se na dificuldade em efetuar bioensaios e no fato de a maioria dos laboratórios não dispor de calorímetro. Nesse caso, trabalhar com equações geradas a partir de análises químicas simples pode auxiliar o nutricionista. Além disso, mesmo que os laboratórios tenham calorímetro, a execução de ensaios biológicos é dispendiosa e demorada.

Segundo Sakamura e Silva (1998), vários pesquisadores desenvolveram equações de predição para estimar o conteúdo de energia dos ingredientes com base na composição química.

A composição dos subprodutos do trigo tem apresentado grande variação. A maioria dos moinhos combina todos os componentes, resultando na diferença entre o material produzido, o que dificulta estabelecer valores energéticos adequados para os derivados do trigo (Sakamura, Silva, 1998).

O Nutrient... (1994) apresenta várias equações de predição de energia metabolizável aparente corrigida pelo nitrogênio $\left(\mathrm{EMA}_{\mathrm{n}}\right)$ para vários alimentos, a partir da composição química, propostas por diversos autores. A equação proposta para o trigo e seus subprodutos sob a forma de farelos e farinhas é a seguinte: $\mathrm{EMA}_{\mathrm{n}}=3,985-205 \mathrm{FB}$. Outra equação é proposta para o trigo e seus subprodutos quando na 
Equações de regressão para estimar valores energéticos...

forma peletizada: $\mathrm{EMA}_{\mathrm{n}}=3,926$-185 FB. A proposição das duas equações leva a crer que existe efeito da apresentação do alimento sobre a determinação da energia metabolizável.

O objetivo deste trabalho, a partir dos resultados encontrados em ensaios biológicos e da análise química dos alimentos, foi o de estimar equações para a predição da energia metabolizável (EMA, EMA, EMV e $\mathrm{EMV}_{\mathrm{n}}$ ) do trigo e seus produtos.

\section{MATERIAL E MÉTODOS}

Foram desenvolvidas equações de predição para a energia metabolizável baseando-se nas análises bromatológicas dos alimentos e da ração-referência e nos valores de energia metabolizável aparente (EMA), energia metabolizável verdadeira (EMV), energia metabolizável aparente corrigida pelo nitrogênio $\left(E M A_{n}\right)$ e energia metabolizável verdadeira corrigida pelo nitrogênio $\left(E M V_{n}\right)$, obtidas em cinco tratamentos utilizando diferentes metodologias de avaliação (Tab. 1). 


\section{Borges et al.}

Tabela 1. Valores de energia metabolizável (kcal/kg) da matéria seca, segundo o alimento e a metodologia experimental

\begin{tabular}{|c|c|c|c|c|c|}
\hline \multirow{2}{*}{ Alimento $^{1}$} & \multicolumn{3}{|c|}{ Metodologia tradicional } & \multicolumn{2}{|c|}{ limentação forçada } \\
\hline & Trat. 1 & Trat. 2 & Trat. 3 & Trat. 4 & Trat. 5 \\
\hline & \multicolumn{5}{|c|}{ Energia metabolizável aparente - EMA } \\
\hline $\mathrm{RR}$ & 3351 & 3384 & 2357 & 2170 & 2704 \\
\hline TIM & 4221 & 4114 & 3217 & 2932 & 3330 \\
\hline GT & 3006 & 2948 & 2086 & 1991 & 2627 \\
\hline FTC & 4046 & 4025 & 3156 & 2783 & 3203 \\
\hline FTE & 3907 & 3934 & 2830 & 2527 & 3243 \\
\hline FTH & 1686 & 1706 & 949 & 1013 & 1510 \\
\hline FTA & 1931 & 2097 & 1108 & 1250 & 1626 \\
\hline \multirow[t]{2}{*}{ FTG } & 2028 & 2223 & 1336 & 1170 & 1677 \\
\hline & \multicolumn{5}{|c|}{ Energia metabolizável verdadeira - EMV } \\
\hline $\mathrm{RR}$ & 3417 & 3416 & 3341 & 3388 & 3371 \\
\hline TIM & 4273 & 4137 & 4224 & 4179 & 3983 \\
\hline GT & 3094 & 2971 & 3068 & 3206 & 3292 \\
\hline FTC & 4113 & 4066 & 4156 & 4022 & 3881 \\
\hline FTE & 3971 & 3962 & 3820 & 3753 & 3915 \\
\hline FTH & 1767 & 1737 & 1927 & 2223 & 2173 \\
\hline FTA & 2002 & 2120 & 2084 & 2459 & 2289 \\
\hline \multirow[t]{2}{*}{ FTG } & 2107 & 2250 & 2329 & 2400 & 2351 \\
\hline & \multicolumn{5}{|c|}{ Energia metabolizável aparente corrigida pelo nitrogênio - EMAn } \\
\hline $\mathrm{RR}$ & 3121 & 3200 & 2084 & 1785 & 2433 \\
\hline TIM & 4059 & 4001 & 3003 & 2728 & 3155 \\
\hline GT & 2758 & 2785 & 1846 & 1620 & 2310 \\
\hline FTC & 3898 & 3881 & 2838 & 2683 & 3022 \\
\hline FTE & 3706 & 3754 & 2576 & 2329 & 3014 \\
\hline FTH & 1557 & 1614 & 860 & 710 & 1295 \\
\hline FTA & 1789 & 1938 & 982 & 718 & 1462 \\
\hline \multirow[t]{2}{*}{ FTG } & 1911 & 2105 & 1148 & 863 & 1522 \\
\hline & \multicolumn{5}{|c|}{ Energia metabolizável verdadeira corrigida pelo nitrogênio - EMVn } \\
\hline $\mathrm{RR}$ & 3187 & 3231 & 3067 & 3004 & 3100 \\
\hline TIM & 4111 & 4024 & 4010 & 3976 & 3838 \\
\hline GT & 2846 & 2809 & 2828 & 2835 & 2976 \\
\hline FTC & 3966 & 3922 & 3839 & 3923 & 3701 \\
\hline FTE & 3770 & 3782 & 3566 & 3556 & 3686 \\
\hline FTH & 1639 & 1646 & 1838 & 1921 & 1958 \\
\hline FTA & 1860 & 2006 & 1958 & 1927 & 2125 \\
\hline FTG & 1990 & 2132 & 2141 & 2093 & 2196 \\
\hline
\end{tabular}

1: $\mathrm{RR}=$ ração-referência, TIM= trigo integral moído, GT= germe de trigo, FTC= farinha de trigo clara, FTE= farinha de trigo escura, $\mathrm{FTH}=$ farelo de trigo para uso humano, FTA= farelo de trigo para uso animal, FTG= farelo de trigo grosso.

Trat.1- Metodologia tradicional. Alimentação à vontade e coleta total de excreta com pintos de 14 dias de idade

Trat.2- Metodologia tradicional. Alimentação à vontade e coleta total de excreta com frangos de 39 dias de idade

Trat.3- Metodologia de alimentação forçada - 25 gramas de alimento e aves intactas

Trat.4 - Metodologia de alimentação forçada - 25 gramas de alimento e aves cecectomizadas

Trat.5 - Metodologia de alimentação forçada - 50 gramas de alimento e aves cecectomizadas

As variáveis utilizadas para os cálculos das equações foram os valores de matéria seca (MS), proteína bruta (PB), fibra bruta (FB), fibra em detergente neutro (FDN), fibra em detergente ácido (FDA), estrato etéreo (EE), cinzas (Cz) e amido dos alimentos (Tab. 2). 
Equações de regressão para estimar valores energéticos...

Tabela 2. Análises químicas (\%) e valores de energia bruta (kcal/g) segundo o alimento utilizado

\begin{tabular}{lccccccccc}
\hline Alimento $^{1}$ & MS & EB & PB & FDN & FDA & FB & Cz & Amido & EE \\
RR & 88,84 & 4521,2 & 25,74 & 15,35 & 0,56 & 3,06 & 6,60 & 47,25 & 3,51 \\
TIM & 86,76 & 5068,3 & 22,10 & 16,43 & 1,73 & 1,84 & 1,68 & 56,24 & 1,90 \\
GT & 89,05 & 4333,6 & 32,02 & 8,39 & ND & ND & 4,95 & 53,43 & 10,4 \\
FTC & 87,34 & 4654,5 & 14,13 & 6,64 & ND & ND & 0,56 & 87,54 & 1,02 \\
FTE & 88,22 & 4508,2 & 18,33 & 4,73 & ND & 0,48 & 1,77 & 82,71 & 3,24 \\
FTH & 89,39 & 4277,4 & 19,63 & 37,03 & 6,82 & 7,71 & 4,97 & 32,47 & 5,12 \\
FTA & 89,50 & 4488,7 & 18,07 & 44,25 & 9,53 & 10,12 & 5,58 & 30,30 & 4,07 \\
FTG & 87,99 & 4681,8 & 18,50 & 41,68 & 7,22 & 8,40 & 5,85 & 32,38 & 4,04 \\
\hline
\end{tabular}

Dados expressos na base da matéria seca.

1: $\mathrm{RR}=$ ração-referência, TIM= trigo integral moído, GT= germe de trigo, FTC= farinha de trigo clara, FTE= farinha de trigo escura, $\mathrm{FTH}=$ farelo de trigo para uso humano, FTA= farelo de trigo para uso animal, FTG= farelo de trigo grosso. MS= matéria seca, $\mathrm{EB}=$ energia bruta, $\mathrm{PB}=$ proteína bruta, FDN= fibra em detergente neutro, FDA= fibra em detergente ácido, FB = fibra bruta, $\mathrm{Cz}=$ cinzas, $\mathrm{EE}=$ extrato etéreo. $\mathrm{ND}=$ não detectado pelos limites da técnica.

Para as equações foram utilizadas as análises químicas e os dados de EM de todos os tratamentos ou agrupados por metodologia ou nível de consumo, aplicando-se o stepwise. As equações foram obtidas em agrupamentos de acordo com a metodologia para a obtenção da EM, divididas em cinco grupos: valores de EM de todos os tratamentos, sem considerar o efeito da metodologia ou nível de consumo (tratamentos 1, 2, 3, 4 e 5); valores de EM dos tratamentos com metodologia tradicional (tratamentos 1 e 2); valores de EM dos tratamentos com metodologia de alimentação forçada (tratamentos 3, 4 e 5); valores de EM dos tratamentos com metodologia de alimentação forçada e consumo igual (tratamentos 3 e 4); valores de EM dos tratamentos com metodologia de alimentação forçada e consumo diferente (tratamentos 4 e 5) descritos por Borges et al. (2003).

\section{RESULTADOS E DISCUSSÃO}

Ainda que todos os alimentos utilizados neste trabalho sejam derivados do trigo, eles apresentam composição nutricional bastante distinta um dos outros. Isso permite que se desenvolvam equações de predição baseadas nessas análises. Na Tab. 3 apresentam-se as equações de predição para os valores de energia metabolizável aparente a partir das análises de FB, EE, PB, Cz e amido. A FB foi a variável que melhor se relacionou (negativamente) com os valores de energia metabolizável. Esse resultado já era esperado pois, segundo Carré et al. (1984), a parede celular dos alimentos é considerada um diluidor da energia metabolizável dos alimentos. Entretanto, o valor de FB isolado não foi suficiente para uma boa estimativa dos valores energéticos, com $\mathrm{R}^{2}$ abaixo de 0,80 .

Quando a FB foi somada ao EE aumentou-se a precisão das equações, com valores de $\mathrm{R}^{2}$ acima de 0,80 na maioria das equações. A entrada de outras variáveis como $\mathrm{PB}, \mathrm{Cz}$ e amido não melhorou significativamente os $\mathrm{R}^{2}$ das equações.

Com a retirada da FB das equações, todas as outras variáveis somadas originaram um $\mathrm{R}^{2}$ próximo ao encontrado com as variáveis FB e EE. Sibbald e Price (1977), ao estimarem as correlações entre as características químicas e físicas do trigo com a EMA, verificaram que o fósforo e os CHO solúveis foram as características químicas que melhor se correlacionaram com a energia, enquanto que FB e EE não apresentaram correlações significativas.

Os cálculos a partir dos valores de todos os tratamentos, sem considerar o efeito da metodologia, resultaram em equações menos precisas para EMA, com os menores $\mathrm{R}^{2}$. Isto se deveu aos efeitos dos valores obtidos nos tratamentos de alimentação forçada, fortemente afetados pelo nível de consumo. 


\section{Borges et al.}

Tabela 3. Equações de predição da energia metabolizável aparente (EMA) a partir de análises químicas dos alimentos

\begin{tabular}{|c|c|c|c|}
\hline Análise química & Equação de predição & GL & $\mathrm{R}^{2}$ \\
\hline \multicolumn{4}{|c|}{ FB } \\
\hline Todos os tratamentos & $\mathrm{EMA}=3271,3-186,5 \mathrm{FB}$ & 38 & 0,59 \\
\hline Metodologia tradicional & $\mathrm{EMA}=3853,4-206,3 \mathrm{FB}$ & 14 & 0,75 \\
\hline Todos os Sibbald & $\mathrm{EMA}=2883,2-173,2 \mathrm{FB}$ & 22 & 0,72 \\
\hline Sibbald-consumo igual & $\mathrm{EMA}=2745,5-174,8 \mathrm{FB}$ & 14 & 0,74 \\
\hline Sibbald-consumo distinto & $\mathrm{EMA}=2881,3-164,1 \mathrm{FB}$ & 14 & 0,70 \\
\hline \multicolumn{4}{|c|}{$\mathrm{FB}+\mathrm{EE}$} \\
\hline Todos os tratamentos & $\mathrm{EMA}=3821,7-188,4 \mathrm{FB}-129,8 \mathrm{EE}$ & 37 & 0,72 \\
\hline Metodologia tradicional & $\mathrm{EMA}=4498,3-208,6 \mathrm{FB}-152,0 \mathrm{EE}$ & 13 & 0,93 \\
\hline Todos os Sibbald & $\mathrm{EMA}=3370,6-174,9 \mathrm{FB}-114,9 \mathrm{EE}$ & 21 & 0,86 \\
\hline Sibbald-consumo igual & $\mathrm{EMA}=3284,3-176,7 \mathrm{FB}-127,0 \mathrm{EE}$ & 13 & 0,92 \\
\hline Sibbald-consumo distinto & $\mathrm{EMA}=3310,1-165,6 \mathrm{FB}-101,0 \mathrm{EE}$ & 13 & 0,84 \\
\hline \multicolumn{4}{|c|}{$\mathrm{FB}+\mathrm{EE}+\mathrm{PB}$} \\
\hline Todos os tratamentos & $\mathrm{EMA}=3204,8-174,0 \mathrm{FB}-188,4 \mathrm{EE}+38,2 \mathrm{~PB}$ & 36 & 0,73 \\
\hline Metodologia tradicional & $\mathrm{EMA}=3770,8-191,7 \mathrm{FB}-221,3 \mathrm{EE}+45,1 \mathrm{~PB}$ & 12 & 0,95 \\
\hline Todos os Sibbald & $\mathrm{EMA}=2828,0-162,3 \mathrm{FB}-166,5 \mathrm{EE}+33,6 \mathrm{~PB}$ & 20 & 0,88 \\
\hline Sibbald-consumo igual & $\mathrm{EMA}=2704,0-163,2 \mathrm{FB}-182,2 \mathrm{EE}+34,9 \mathrm{~PB}$ & 12 & 0,94 \\
\hline Sibbald-consumo distinto & $\mathrm{EMA}=2781,5-153,3 \mathrm{FB}-151,3 \mathrm{EE}+32,7 \mathrm{~PB}$ & 12 & 0,84 \\
\hline \multicolumn{4}{|c|}{$\mathrm{FB}+\mathrm{EE}+\mathrm{PB}+\mathrm{Cz}$} \\
\hline Todos os tratamentos & $\mathrm{EMA}=2759,0-123,2 \mathrm{FB}-194,4 \mathrm{EE}+71,6 \mathrm{~PB}-108,5 \mathrm{Cz}$ & 35 & 0.74 \\
\hline Metodologia tradicional & EMA = Não estimada pelo método & - & - \\
\hline Todos os Sibbald & $\mathrm{EMA}=2363,7-109,3 \mathrm{FB}-172,7 \mathrm{EE}+68,4 \mathrm{~PB}-113,0 \mathrm{Cz}$ & 19 & 0,89 \\
\hline Sibbald-consumo igual & $\mathrm{EMA}=2184,3-103,9 \mathrm{FB}-189,2 \mathrm{EE}+74,9 \mathrm{~PB}-126,5 \mathrm{Cz}$ & 11 & 0,96 \\
\hline Sibbald-consumo distinto & $\mathrm{EMA}=2323,9-101,1 \mathrm{FB}-157,5 \mathrm{EE}+67,1 \mathrm{~PB}-111,5 \mathrm{Cz}$ & 11 & 0,86 \\
\hline \multicolumn{4}{|c|}{$\mathrm{FB}+\mathrm{EE}+\mathrm{PB}+\mathrm{Cz}+\mathrm{A}$} \\
\hline Todos os trat & $\mathrm{EMA}=-1769,5+92,6 \mathrm{FB}-236,4 \mathrm{EE}+174,6 \mathrm{~PB}-151$ & 34 & 0,76 \\
\hline Metodologia tradicional & $\mathrm{EMA}=-2460,7+133,0 \mathrm{FB}-280,8 \mathrm{EE}+208,7 \mathrm{~PB}-156,3 \mathrm{Cz}+45,0 \mathrm{~A}$ & 10 & 0,96 \\
\hline Todos os Sibbald & $\mathrm{EMA}=-1308,7+65,6 \mathrm{FB}-206,8 \mathrm{EE}+151,9 \mathrm{~PB}-147,5 \mathrm{Cz}+28,4 \mathrm{~A}$ & 18 & 0,90 \\
\hline Sibbald-consumo igual & $\mathrm{EMA}=-910,8+43,5 \mathrm{FB}-217,9 \mathrm{EE}+145,3 \mathrm{~PB}-155,6 \mathrm{CZ}+43,3 \mathrm{~A}$ & 10 & 0,96 \\
\hline Sibbald-consumo distinto & $\mathrm{EMA}=-1918,2+101,0 \mathrm{FB}-196,9 \mathrm{EE}+163,5 \mathrm{~PB}-151,2 \mathrm{Cz}+32,8 \mathrm{~A}$ & 10 & 0,88 \\
\hline \multicolumn{4}{|c|}{$\mathrm{EE}+\mathrm{PB}+\mathrm{Cz}+\mathrm{A}$} \\
\hline Todos os tratamentos & $\mathrm{EMA}=92,2-217,8 \mathrm{EE}+129,3 \mathrm{~PB}-122,5 \mathrm{Cz}+21,1 \mathrm{~A}$ & 35 & 0,75 \\
\hline Metodologia tradicional & $\mathrm{EMA}=213,9-254,1 \mathrm{EE}+143,6 \mathrm{~PB}-115,4 \mathrm{CZ}+25,0 \mathrm{~A}$ & 11 & 0,98 \\
\hline Todos os Sibbald & $\mathrm{EMA}=11,1-193,6 \mathrm{EE}+119,8 \mathrm{~PB}-127,3 \mathrm{Cz}+18,5 \mathrm{~A}$ & 19 & 0,90 \\
\hline Sibbald-consumo igual & $\mathrm{EMA}=-34,5-209,1 \mathrm{EE}+124,0 \mathrm{~PB}-142,2 \mathrm{Cz}+124,0 \mathrm{~A}$ & 11 & 0,97 \\
\hline Sibbald-consumo distinto & $\mathrm{EMA}=114,1-176,5 \mathrm{EE}+114,1 \mathrm{~PB}-120,1 \mathrm{Cz}+17,6 \mathrm{~A}$ & 11 & 0,87 \\
\hline
\end{tabular}

As equações derivadas dos valores obtidos nos tratamentos com metodologia tradicional apresentaram os maiores valores de $\mathrm{R}^{2}$ sendo, portanto, mais adequadas para predizer a energia metabolizável aparente dos alimentos. Da mesma forma, quando se utilizaram os dados gerados apenas pelos tratamentos da alimentação forçada, obtiveram-se regressões igualmente precisas.

No caso da divisão dos tratamentos de alimentação forçada, as equações geradas a partir dos dados obtidos nos tratamentos com consumo igual, embora um deles fosse com aves cecectomizadas e outro com aves normais, apresentaram o $\mathrm{R}^{2}$ maior do que aquelas com consumo distinto, ambos com aves cecectomizadas.

Na Tab. 4 apresentam-se as equações de predição dos valores de energia metabolizável aparente corrigida a partir das análises de FB, EE, PB, Cz e amido. A correção da energia metabolizável pelo nitrogênio não melhorou a precisão das equações, ao contrário, todas as equações apresentaram $\mathrm{R}^{2}$ inferiores ou iguais àquelas para a predição de EMA sem correção. 
Equações de regressão para estimar valores energéticos...

Tabela 4. Equações de predição da energia metabolizável aparente corrigida $\left(\mathrm{EMA}_{\mathrm{n}}\right)$ a partir de análises químicas dos alimentos

\begin{tabular}{|c|c|c|c|}
\hline Análise química & Equação de predição & GL & $\mathrm{R}^{2}$ \\
\hline \multicolumn{4}{|c|}{ FB } \\
\hline Todos os tratamentos & EMA $_{n}=3052,9-183,5$ FB & 38 & 0,54 \\
\hline Metodologia tradicional & $\mathrm{EMA}_{\mathrm{n}}=3671,7-199,7 \mathrm{FB}$ & 14 & 0,73 \\
\hline Todos os Sibbald & $\mathrm{EMA}_{\mathrm{n}}=2640,4-172,7 \mathrm{FB}$ & 22 & 0,69 \\
\hline Sibbald-consumo igual & $\mathrm{EMA}_{\mathrm{n}}=2499,1-177,3 \mathrm{FB}$ & 14 & 0,73 \\
\hline Sibbald-consumo distinto & $\mathrm{EMA}_{\mathrm{n}}=2653,6-170,9 \mathrm{FB}$ & 14 & 0,66 \\
\hline \multicolumn{4}{|c|}{$\mathrm{FB}+\mathrm{EE}$} \\
\hline Todos os tratamentos & $\mathrm{EMA}_{n}=3639,2-185,6 \mathrm{FB}-138,2 \mathrm{EE}$ & 37 & 0,69 \\
\hline Metodologia tradicional & $\mathrm{EMA}_{\mathrm{n}}=4337,0-202,0 \mathrm{FB}-156,8 \mathrm{EE}$ & 13 & 0,93 \\
\hline Todos os Sibbald & $\mathrm{EMA}_{\mathrm{n}}=3173,9-174,6 \mathrm{FB}-125,8 \mathrm{EE}$ & 21 & 0,85 \\
\hline Sibbald-consumo igual & $\mathrm{EMA}_{\mathrm{n}}=3074,7-179,5 \mathrm{FB}-135,7 \mathrm{EE}$ & 13 & 0,92 \\
\hline Sibbald-consumo distinto & $\mathrm{EMA}_{n}=3165,5-172,8 \mathrm{FB}-120,7 \mathrm{EE}$ & 13 & 0,82 \\
\hline \multicolumn{4}{|c|}{$\mathrm{FB}+\mathrm{EE}+\mathrm{PB}$} \\
\hline Todos os tratamentos & $\mathrm{EMA}_{n}=3106,9-173,2 \mathrm{FB}-188,8 \mathrm{EE}+32,9$ PB & 36 & 0,70 \\
\hline Metodologia tradicional & $\mathrm{EMA}_{n}=3670,5-186,6 \mathrm{FB}-220,9 \mathrm{EE}+41,3 \mathrm{~PB}$ & 12 & 0,95 \\
\hline Todos os Sibbald & $\mathrm{EMA}_{n}=2731,3-164,3 \mathrm{FB}-167,9 \mathrm{EE}+27,5 \mathrm{~PB}$ & 20 & 0,86 \\
\hline Sibbald-consumo igual & $\mathrm{EMA}_{n}=2620,6-168,6 \mathrm{FB}-178,9 \mathrm{EE}+28,2 \mathrm{~PB}$ & 12 & 0,94 \\
\hline Sibbald-consumo distinto & $\mathrm{EMA}_{n}=2778,9-163,8 \mathrm{FB}-157,5 \mathrm{EE}+23,9 \mathrm{~PB}$ & 12 & 0,82 \\
\hline \multicolumn{4}{|c|}{$\mathrm{FB}+\mathrm{EE}+\mathrm{PB}+\mathrm{Cz}$} \\
\hline Todos os tratamentos & $\mathrm{EMA}_{\mathrm{n}}=2573,9-112,4 \mathrm{FB}-196,0 \mathrm{EE}+72,9 \mathrm{~PB}-129,8 \mathrm{Cz}$ & 35 & 0,71 \\
\hline Metodologia tradicional & $\mathrm{EMA}_{\mathrm{n}}=3183,2-130,9 \mathrm{FB}-226,8 \mathrm{EE}+77,8 \mathrm{~PB}-118,7 \mathrm{Cz}$ & 11 & 0,96 \\
\hline Todos os Sibbald & $\mathrm{EMA}_{n}=2167,7-100,0 \mathrm{FB}-175,5 \mathrm{EE}+69,7 \mathrm{~PB}-137,3 \mathrm{Cz}$ & 19 & 0,88 \\
\hline Sibbald-consumo igual & $\mathrm{EMA}_{\mathrm{n}}=2005,9-98,7 \mathrm{FB}-187,2 \mathrm{EE}+74,2 \mathrm{~PB}-149,7 \mathrm{Cz}$ & 11 & 0,96 \\
\hline Sibbald-consumo distinto & $\mathrm{EMA}_{n}=2233,9-101,6 \mathrm{FB}-164,8 \mathrm{EE}+64,9 \mathrm{~PB}-132,8 \mathrm{Cz}$ & 11 & 0,84 \\
\hline \multicolumn{4}{|c|}{$\mathrm{FB}+\mathrm{EE}+\mathrm{PB}+\mathrm{Cz}+\mathrm{A}$} \\
\hline Todos os tratamentos & $\mathrm{EMA}_{n}=-1182,2+66,7 \mathrm{FB}-230,9 \mathrm{EE}+158,4 \mathrm{~PB}-165,0 \mathrm{Cz}+29,0 \mathrm{~A}$ & 34 & 0,72 \\
\hline Metodologia tradicional & $\mathrm{EMA}_{\mathrm{n}}=-1998,7+115,9 \mathrm{~B}-274,9 \mathrm{EE}+195,8 \mathrm{~PB}-167,3 \mathrm{Cz}+40,1 \mathrm{~A}$ & 10 & 0,96 \\
\hline Todos os Sibbald & $\mathrm{EMA}_{\mathrm{n}}=-637,9+33,7 \mathrm{FB}-201,6 \mathrm{EE}+133,5 \mathrm{~PB}-163,6 \mathrm{Cz}+21,7 \mathrm{~A}$ & 18 & 0,89 \\
\hline Sibbald-consumo igual & $\mathrm{EMA}_{\mathrm{n}}=100,5-7,9 \mathrm{FB}-204,9 \mathrm{EE}+117,6 \mathrm{~PB}-167,6 \mathrm{Cz}+14,8 \mathrm{~A}$ & 10 & 0,96 \\
\hline Sibbald-consumo distinto & $\mathrm{EMA}_{\mathrm{n}}=-773,5+41,8 \mathrm{FB}-192,7 \mathrm{EE}+133,2 \mathrm{~PB}-160,98 \mathrm{Cz}+23,3 \mathrm{~A}$ & 10 & 0,85 \\
\hline \multicolumn{4}{|c|}{$\mathrm{EE}+\mathrm{PB}+\mathrm{Cz}+\mathrm{A}$} \\
\hline Todos os tratamentos & $\mathrm{EMA}_{\mathrm{n}}=157,1-217,6 \mathrm{EE}+125,9 \mathrm{~PB}-144,7 \mathrm{Cz}+19,1 \mathrm{~A}$ & 35 & 0,72 \\
\hline Metodologia tradicional & $\mathrm{EMA}_{\mathrm{n}}=333,6-251,6 \mathrm{EE}+138,99 \mathrm{~PB}-131,7 \mathrm{Cz}+22,8 \mathrm{~A}$ & 11 & 0,98 \\
\hline Todos os Sibbald & $\mathrm{EMA}_{\mathrm{n}}=39,5-194,8 \mathrm{EE}+117,03 \mathrm{~PB}-153,3 \mathrm{Cz}+16,7 \mathrm{~A}$ & 19 & 0,89 \\
\hline Sibbald-consumo igual & $\mathrm{EMA}_{\mathrm{n}}=-57,9-206,5 \mathrm{EE}+121,5 \mathrm{~PB}-170,0 \mathrm{Cz}+15,9 \mathrm{~A}$ & 11 & 0,97 \\
\hline Sibbald-consumo distinto & $\mathrm{EMA}_{\mathrm{n}}=66,3-184,4 \mathrm{EE}+112,9 \mathrm{~PB}-148,2 \mathrm{Cz}+17,02 \mathrm{~A}$ & 11 & 0,85 \\
\hline
\end{tabular}

Dale (1996), citado por Sakamura e Silva (1998), encontrou a seguinte equação: $\mathrm{EMA}_{\mathrm{n}}=3086-165 \times$ FB e $\mathrm{R}^{2}=0,77$ ao trabalhar com subprodutos de trigo, baseando-se em dados de literatura. O coeficiente linear da equação obtida pelo autor aproxima-se mais daqueles obtidos com os dados de todos os tratamentos (3052,9-183,5 FB e $\mathrm{R}^{2}$ de 0,59) e tratamentos com metodologia tradicional (3671,7-199,7 FB) deste trabalho. Entretanto, o $\mathrm{R}^{2}$ da equação de Dale foi mais compatível com a equação obtida a partir dos dados gerados por tratamentos da metodologia tradicional e da alimentação forçada com consumo igual. De qualquer forma, o $\mathrm{R}^{2}$ encontrado pelo autor para essa equação foi mais alto que todos os encontrados neste trabalho para equações semelhantes.

Semelhante ao ocorrido com a EMA, a FB foi a variável que melhor explicou os valores de $\mathrm{EMA}_{\mathrm{n}}$, embora sozinha não tenha sido um bom preditor, com $\mathrm{R}^{2}$ de 0,54 , quando a equação foi obtida a partir de dados gerados por todos os tratamentos. Resultados semelhantes foram encontrados por Campbell et al. (1986), que relataram ser a FB e o EE as variáveis que determinaram a maior precisão das equações para a predição da $\mathrm{EMA}_{n}$. Os demais resultados foram semelhantes aos obtidos para as equações de predição de EMA. 


\section{Borges et al.}

Era de se esperar que o EE, por seu alto conteúdo de fibra, apresentasse sempre relações positivas com o aumento energético nas equações. Entretanto, em quase todas elas o EE apresenta relação negativa. Fisiologicamente não há uma explicação plausível para essa relação, porém, matematicamente, esse fato pode ser justificado pelos altos valores atribuídos ao intercepto da equação. Equações em que o EE se correlaciona negativamente com o aumento de energia também foram encontradas por Albino (1980)

Do mesmo modo, fisiologicamente e nutricionalmente não é possível explicar as correlações positivas da FB em algumas equações, embora se observe que em todas as equações em que o fato ocorreu, foi atribuído um valor negativo ao intercepto da equação, o que justifica, matematicamente, a inversão de sinais.

As equações de predição da EMV e EMV a partir das análises de FB, EE, PB, CZ e amido encontram-se nas Tab. 5 e 6. Novamente, FB foi a variável que melhor explicou os resultados tanto para EMV como para $\mathrm{EMV}_{\mathrm{n}}$. Independente da utilização de todos os dados experimentais ou seu agrupamento por metodologia, todas as equações apresentaram $\mathrm{R}^{2}$ bem próximos, variando de 0,74 a 0,76 , mostrando que, eliminadas as diferenças causadas pelo consumo, as equações não são mais influenciadas pelo efeito provocado pela metodologia. 
Equações de regressão para estimar valores energéticos...

Tabela 5. Equações de predição da energia metabolizável verdadeira (EMV) a partir de análises químicas dos alimentos

\begin{tabular}{|c|c|c|c|}
\hline Análise química & Equação de predição & GL & $\mathrm{R}^{2}$ \\
\hline \multicolumn{4}{|c|}{ FB } \\
\hline Todos os tratamentos & $\mathrm{EMV}=3870,7-187,2 \mathrm{FB}$ & 38 & 0,75 \\
\hline Metodologia tradicional & $\mathrm{EMV}=3902,5-206,2 \mathrm{FB}$ & 14 & 0,75 \\
\hline Todos os Sibbald & $\mathrm{EMV}=3849,4-174,5 \mathrm{FB}$ & 22 & 0,77 \\
\hline Sibbald-consumo igual & $\mathrm{EMV}=3857,8-176,2 \mathrm{FB}$ & 14 & 0,74 \\
\hline Sibbald-consumo distinto & $\mathrm{EMV}=3833,8-165,3 \mathrm{FB}$ & 14 & 0,79 \\
\hline \multicolumn{4}{|c|}{$\mathrm{FB}+\mathrm{EE}$} \\
\hline Todos os tratamentos & $\mathrm{EMV}=4425,2-189,1 \mathrm{FB}-130,7 \mathrm{EE}$ & 37 & 0,92 \\
\hline Metodologia tradicional & $\mathrm{EMV}=4542,6-208,4 \mathrm{FB}-150,9 \mathrm{EE}$ & 13 & 0,94 \\
\hline Todos os Sibbald & $\mathrm{EMV}=4346,8-176,2 \mathrm{FB}-117,2 \mathrm{EE}$ & 21 & 0,93 \\
\hline Sibbald-consumo igual & EMV $=4408,1-178,2$ FB $-129,7 \mathrm{EE}$ & 13 & 0,92 \\
\hline Sibbald-consumo distinto & $\mathrm{EMV}=4272,4-166,9 \mathrm{FB}-103,4 \mathrm{EE}$ & 13 & 0,93 \\
\hline \multicolumn{4}{|c|}{$\mathrm{FB}+\mathrm{EE}+\mathrm{PB}$} \\
\hline Todos os tratamentos & $\mathrm{EMV}=3813,0-174,9 \mathrm{FB}-188,9 \mathrm{EE}+37,9 \mathrm{~PB}$ & 36 & 0,94 \\
\hline Metodologia tradicional & $\mathrm{EMV}=3830,5-191,8 \mathrm{FB}-218,6 \mathrm{EE}+44,1 \mathrm{~PB}$ & 12 & 0,95 \\
\hline Todos os Sibbald & $\mathrm{EMV}=3801,3-163,5 \mathrm{FB}-169,1 \mathrm{EE}+33,8 \mathrm{~PB}$ & 20 & 0,94 \\
\hline Sibbald-consumo igual & $\mathrm{EMV}=3824,5-164,6 \mathrm{FB}-185,2 \mathrm{EE}+36,1 \mathrm{~PB}$ & 12 & 0,94 \\
\hline Sibbald-consumo distinto & $\mathrm{EMV}=3740,9-154,5 \mathrm{FB}-153,9 \mathrm{EE}+32,9 \mathrm{~PB}$ & 12 & 0,95 \\
\hline \multicolumn{4}{|c|}{$\mathrm{FB}+\mathrm{EE}+\mathrm{PB}+\mathrm{Cz}$} \\
\hline Todos os tratamentos & $\mathrm{EMV}=3363,6-123,6 \mathrm{FB}-195,0 \mathrm{EE}+71,6 \mathrm{~PB}-109,4 \mathrm{Cz}$ & 35 & 0,95 \\
\hline Metodologia tradicional & EMV = Não estimado pelo método & - & - \\
\hline Todos os Sibbald & $\mathrm{EMV}=322,1-108,8 \mathrm{FB}-75,6 \mathrm{EE}+69,7 \mathrm{~PB}-116,7 \mathrm{Cz}$ & 19 & 0,96 \\
\hline Sibbald-consumo igual & $\mathrm{EMV}=3287,6-103,3 \mathrm{FB}+192,4 \mathrm{EE}+76,4 \mathrm{~PB}-130,7 \mathrm{Cz}$ & 11 & 0,96 \\
\hline Sibbald-consumo distinto & $\mathrm{EMV}=3268,6-100,6 \mathrm{FB}-160,3 \mathrm{EE}+68,3 \mathrm{~PB}-115,0 \mathrm{Cz}$ & 11 & 0,97 \\
\hline \multicolumn{4}{|c|}{$\mathrm{FB}+\mathrm{EE}+\mathrm{PB}+\mathrm{Cz}+\mathrm{A}$} \\
\hline Todos os tratamentos & $\mathrm{EMV}=-1043,5+86,4 \mathrm{FB}-235,9 \mathrm{EE}+171,8 \mathrm{~PB}-150,8 \mathrm{Cz}$ & 34 & 0,97 \\
\hline Metodologia tradicional & $\mathrm{EMV}=-2264,9+125,5 \mathrm{FB}-276,9 \mathrm{EE}+203,9 \mathrm{~PB}-151,9 \mathrm{Cz}+44,0 \mathrm{~A}$ & 10 & 0,98 \\
\hline Todos os Sibbald & $\mathrm{EMV}=-229,1+60,3 \mathrm{FB}-208,5 \mathrm{EE}+150,5 \mathrm{~PB}-116,7 \mathrm{Cz}+69,7 \mathrm{~A}$ & 18 & 0,96 \\
\hline Sibbald-consumo igual & $\mathrm{EMV}=-332,0+37,5 \mathrm{FB}-219,9 \mathrm{EE}+143,6 \mathrm{~PB}-158,5 \mathrm{Cz}+22,8 \mathrm{~A}$ & 10 & 0,97 \\
\hline Sibbald-consumo distinto & $\mathrm{EMV}=-854,0+95,8 \mathrm{FB}-198,5 \mathrm{EE}+162,1 \mathrm{~PB}-153,7 \mathrm{Cz}+31,9 \mathrm{~A}$ & 10 & 0,98 \\
\hline \multicolumn{4}{|c|}{$\mathrm{EE}+\mathrm{PB}+\mathrm{Cz}+\mathrm{A}$} \\
\hline Todos os tratamentos & $\mathrm{EMV}=693,7-218,5 \mathrm{EE}+129,6 \mathrm{~PB}-218,5 \mathrm{Cz}+21,1 \mathrm{~A}$ & 35 & 0,96 \\
\hline Metodologia tradicional & $\mathrm{EMV}=257,8-251,6 \mathrm{EE}+142,5 \mathrm{~PB}-113,3 \mathrm{Cz}+25,2 \mathrm{~A}$ & 11 & 0,98 \\
\hline Todos os Sibbald & $\mathrm{EMV}=984,2-196,4 \mathrm{EE}+121,0 \mathrm{~PB}-131,4 \mathrm{Cz}+18,4 \mathrm{~A}$ & 19 & 0,97 \\
\hline Sibbald-consumo igual & $\mathrm{EMV}=1085,6-212,3 \mathrm{EE}+125,3 \mathrm{~PB}-146,9 \mathrm{Cz}+17,2 \mathrm{~A}$ & 11 & 0,97 \\
\hline Sibbald-consumo distinto & $\mathrm{EMV}=1073,3-179,3 \mathrm{EE}+115,2 \mathrm{~PB}-124,2 \mathrm{Cz}+17,5 \mathrm{~A}$ & 11 & 0,98 \\
\hline
\end{tabular}




\section{Borges et al.}

Tabela 6. Equações de predição da energia metabolizável verdadeira corrigida $\left(\mathrm{EMV}_{\mathrm{n}}\right)$ a partir de análises químicas dos alimentos

\begin{tabular}{|c|c|c|c|}
\hline Análise química & Equação de predição & GL & $\mathrm{R}^{2}$ \\
\hline \multicolumn{4}{|c|}{ FB } \\
\hline Todos os tratamentos & $\mathrm{EMV}_{\mathrm{n}}=3652,3-184,2 \mathrm{FB}$ & 38 & 0,74 \\
\hline Metodologia tradicional & $\mathrm{EMV}_{\mathrm{n}}=3720,9-199,6 \mathrm{FB}$ & 14 & 0,73 \\
\hline Todos os Sibbald & $\mathrm{EMV}_{\mathrm{n}}=3606,7-173,9 \mathrm{FB}$ & 22 & 0,75 \\
\hline Sibbald-consumo igual & $\mathrm{EMV}_{\mathrm{n}}=3611,6-178,8 \mathrm{FB}$ & 14 & 0,74 \\
\hline Sibbald-consumo distinto & $\mathrm{EMV}_{\mathrm{n}}=3606,1-172,2 \mathrm{FB}$ & 14 & 0,76 \\
\hline \multicolumn{4}{|c|}{$\mathrm{FB}+\mathrm{EE}$} \\
\hline Todos os tratamentos & $\mathrm{EMV}_{\mathrm{n}}=4242,7-186,3 \mathrm{FB}-139,2 \mathrm{EE}$ & 37 & 0,93 \\
\hline Metodologia tradicional & $E M V_{n}=4381,4-201,9$ FB - 155,7 EE & 13 & 0,94 \\
\hline Todos os Sibbald & $\mathrm{EMV}_{\mathrm{n}}=4150,3-175,9 \mathrm{FB}-128,2 \mathrm{EE}$ & 21 & 0,94 \\
\hline Sibbald-consumo igual & $\mathrm{EMV}_{\mathrm{n}}=4198,6-180,9 \mathrm{FB}-138,4 \mathrm{EE}$ & 13 & 0,95 \\
\hline Sibbald-consumo distinto & $\mathrm{EMV}_{\mathrm{n}}=4127,8-174,0 \mathrm{FB}-122,9 \mathrm{EE}$ & 13 & 0,94 \\
\hline \multicolumn{4}{|c|}{$\mathrm{FB}+\mathrm{EE}+\mathrm{PB}$} \\
\hline Todos os tratamentos & $\mathrm{EMV}_{\mathrm{n}}=3715,2-174,0 \mathrm{FB}-189,3 \mathrm{EE}+32,7$ PB & 36 & 0,95 \\
\hline Metodologia tradicional & $\mathrm{EMV}_{\mathrm{n}}=3731,0-186,8 \mathrm{FB}-217,6 \mathrm{EE}+40,3 \mathrm{~PB}$ & 12 & 0,95 \\
\hline Todos os Sibbald & $\mathrm{EMV}_{\mathrm{n}}=370,64-165,6 \mathrm{FB}-170,5 \mathrm{EE}+27,6 \mathrm{~PB}$ & 20 & 0,95 \\
\hline Sibbald-consumo igual & $\mathrm{EMV}_{\mathrm{n}}=3741,1-170,25 \mathrm{FB}-181,9 \mathrm{EE}+28,4 \mathrm{~PB}$ & 12 & 0,96 \\
\hline Sibbald-consumo distinto & $\mathrm{EMV}_{\mathrm{n}}=3738,4-164,9 \mathrm{FB}-160,0 \mathrm{EE}+24,1 \mathrm{~PB}$ & 12 & 0,95 \\
\hline \multicolumn{4}{|c|}{$\mathrm{FB}+\mathrm{EE}+\mathrm{PB}+\mathrm{Cz}$} \\
\hline Todos os & $\mathrm{EMV}_{\mathrm{n}}=3178,5-112,8 \mathrm{FB}-196,6 \mathrm{EE}+72,9 \mathrm{~PB}-130,7 \mathrm{Cz}$ & 35 & 0,97 \\
\hline Metodologia tradicional & $\mathrm{EMV}_{\mathrm{n}}=3257,1-132,7 \mathrm{FB}-223,9 \mathrm{EE}+75,8 \mathrm{~PB}-115,4 \mathrm{Cz}$ & 11 & 0,97 \\
\hline Todos os Sibbald & $\mathrm{EMV}_{\mathrm{n}}=3126,1-99,5 \mathrm{FB}-178,4 \mathrm{EE}+70,9 \mathrm{~PB}-178,4 \mathrm{Cz}$ & 19 & 0,98 \\
\hline Sibbald-consumo igual & $\mathrm{EMV}_{\mathrm{n}}=3109,1-98,1 \mathrm{FB}-190,5 \mathrm{EE}+75,7 \mathrm{~PB}-153,9 \mathrm{Cz}$ & 11 & 0,99 \\
\hline Sibbald-consumo distinto & $\mathrm{EMV}_{\mathrm{n}}=3178,6-101,1 \mathrm{FB}-167,6 \mathrm{EE}+66,1 \mathrm{~PB}-136,4 \mathrm{Cz}$ & 11 & 0,98 \\
\hline \multicolumn{4}{|c|}{$\mathrm{FB}+\mathrm{EE}+\mathrm{PB}+\mathrm{Cz}+\mathrm{A}$} \\
\hline Todos os tratamentos & $\mathrm{EMV}_{\mathrm{n}}=-456,2+60,4 \mathrm{FB}-230,3 \mathrm{EE}+155,6 \mathrm{~PB}-164,8 \mathrm{Cz}+28,2 \mathrm{~A}$ & 34 & 0,98 \\
\hline Metodologia tradicional & $\mathrm{EMV}_{\mathrm{n}}=-1803,1+108,5 \mathrm{FB}-270,9 \mathrm{EE}+190,9 \mathrm{~PB}-162,9 \mathrm{Cz}+39,2 \mathrm{~A}$ & 10 & 0,98 \\
\hline Todos os Sibbald & $\mathrm{EMV}_{\mathrm{n}}=441,7+28,4 \mathrm{FB}-203,3 \mathrm{EE}+132,1 \mathrm{~PB}-166,1 \mathrm{Cz}+20,8 \mathrm{~A}$ & 18 & 0,98 \\
\hline Sibbald-consumo igual & $\mathrm{EMV}_{\mathrm{n}}=1343,2-13,9 \mathrm{FB}-206,8 \mathrm{EE}+115,9 \mathrm{~PB}-170,5 \mathrm{Cz}+13,7 \mathrm{~A}$ & 10 & 0,99 \\
\hline Sibbald-consumo distinto & $\mathrm{EMV}_{\mathrm{n}}=290,7+36,5 \mathrm{FB}-194,4 \mathrm{EE}+131,8 \mathrm{~PB}-163,5 \mathrm{Cz}+22,4 \mathrm{~A}$ & 10 & 0,98 \\
\hline \multicolumn{4}{|c|}{$\mathrm{EE}+\mathrm{PB}+\mathrm{Cz}+\mathrm{A}$} \\
\hline Todos os tratamentos & $\mathrm{EMV}_{\mathrm{n}}=758,5-218,2 \mathrm{EE}+126,1 \mathrm{~PB}-146,3 \mathrm{Cz}+19,1 \mathrm{~A}$ & 35 & 0,98 \\
\hline Metodologia tradicional & $\mathrm{EMV}_{\mathrm{n}}=377,5-249,1 \mathrm{EE}+137,9 \mathrm{~PB}-129,6 \mathrm{Cz}+22,9 \mathrm{~A}$ & 11 & 0,98 \\
\hline Todos os Sibbald & $\mathrm{EMV}_{\mathrm{n}}=1012,5-197,6 \mathrm{EE}+118,2 \mathrm{~PB}-157,4 \mathrm{Cz}+16,5 \mathrm{~A}$ & 19 & 0,98 \\
\hline Sibbald-consumo igual & $\mathrm{EMV}_{\mathrm{n}}=1062,2-209,6 \mathrm{EE}+122,7 \mathrm{~PB}-174,8 \mathrm{Cz}+15,8 \mathrm{~A}$ & 11 & 0,99 \\
\hline Sibbald-consumo distinto & $\mathrm{EMV}_{\mathrm{n}}=1025,4-187,1 \mathrm{EE}+113,9 \mathrm{~PB}-152,2 \mathrm{Cz}+16,9 \mathrm{~A}$ & 11 & 0,98 \\
\hline
\end{tabular}

Para o trigo, Sibbald e Price (1977) verificaram que a densidade foi a característica física que melhor se correlacionou com EMV, enquanto que FB, Cz, PB e cálcio foram as características químicas que melhor se correlacionaram com essa medida de energia. Não se observou alta correlação entre EE e EM, segundo os autores. Para a cevada, Sibbald e Price (1976) encontraram altas correlações positivas entre EMV e amido $(0,83)$ ou amido mais açucares $(0,84)$ e negativas entre EMV e FB $(-0,90)$ ou Cz $(-0,76)$.

Para a $\mathrm{EMV}_{\mathrm{n}}$ de subprodutos do trigo, Dale (1996), citado por Sakamura e Silva (1998), encontrou a seguinte equação: $E M V_{n}=3157-116 \times F B$ e $R^{2}=0,67$. O coeficiente linear (a) encontrado por esse autor foi maior do que todos aqueles das equações para predizer $\mathrm{EMV}_{\mathrm{n}}$ com base na $\mathrm{FB}$ encontradas no presente trabalho (Tab. 5) mas, em compensação, a inclinação da curva (b) foi menor. O $\mathrm{R}^{2}$ obtido pelo autor também foi menor que os $\mathrm{R}^{2}$ obtidos neste trabalho para equações semelhantes.

Para as duas incógnitas (EMV e $\left.E M V_{n}\right)$, as equações que melhor as explicaram foram aquelas que

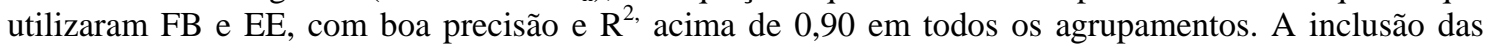
outras variáveis aumentou muito pouco a precisão das equações. 
Para a $E M V_{n}$ pode-se notar, além da alta precisão, que os valores do coeficiente linear da equação são muito próximos, utilizando-se todos os tratamentos ou agrupando-os por metodologia, o que significa pouca influência da metodologia sobre essa variável.

Para todas medidas de energia (EMA, $\mathrm{EMA}_{\mathrm{n}}, \mathrm{EMV}$ e $\mathrm{EMV}_{\mathrm{n}}$ ) a inclusão do amido nas equações não mostrou melhora significativa nas predições. Como a análise de amido é cara e laboriosa, e não faz parte da rotina de muitos laboratórios, a validade das equações baseadas nessa característica química é discutível.

Considerando-se que a FDN apresenta menos erros metodológicos que a FB, era de se esperar que ela se relacionasse melhor com a energia. Entretanto, não foi o que ocorreu, como pode ser observado na Tab. 7, que apresenta as equações de predição para os valores de $E M A, E_{n}, E M V$ e $E_{n} V_{n}$ a partir das análises de FDN e EB.

Tabela 7. Equações de predição da energia metabolizável (EMA, EMA $\left.A_{n}, E M V, E_{n}\right)$ a partir das análises de fibra detergente neutro (FDN) e de energia bruta (EB)

\begin{tabular}{|c|c|c|c|}
\hline Análise química & Equação de predição & GL & $\mathrm{R}^{2}$ \\
\hline \multicolumn{4}{|c|}{ Energia metabolizável aparente FDN } \\
\hline Todos os tratamentos & EMA =3557,3 - 46,8 FDN & 38 & 059 \\
\hline Metodologia tradicional & $\mathrm{EMA}=4174,5-52,0 \mathrm{FDN}$ & 14 & 0,75 \\
\hline Todos os Sibbald & EMA $=3145,8-43,4$ FDN & 22 & 0,72 \\
\hline \multicolumn{4}{|c|}{ Energia metabolizável aparente FDN +EB } \\
\hline Todos os tratamentos & $\mathrm{EMA}=-4039,4-43,8 \mathrm{FDN}+1,6 \mathrm{~EB}$ & 37 & 0,75 \\
\hline Metodologia tradicional & $\mathrm{EMA}=-4511,6-48,6 \mathrm{FDN}+1,8 \mathrm{~EB}$ & 13 & 0,96 \\
\hline Todos os Sibbald & EMA =-3724,7 - 40,7 FDN + 1,5 EB & 21 & 0,90 \\
\hline \multicolumn{4}{|c|}{ Energia metabolizável aparente corrigida FDN } \\
\hline Todos os tratamentos & $\mathrm{EMA}_{\mathrm{n}}=3331,16-46,00 \mathrm{FDN}$ & 38 & 0,54 \\
\hline Metodologia tradicional & $\mathrm{EMA}_{\mathrm{n}}=3979,81-50,30 \mathrm{FDN}$ & 14 & 0,72 \\
\hline Todos os Sibbald & $\mathrm{EMA}_{\mathrm{n}}=2898,73-43,13 \mathrm{FDN}$ & 22 & 0,68 \\
\hline \multicolumn{4}{|c|}{ Energia metabolizável aparente corrigida FDN +EB } \\
\hline Todos os tratamentos & EMA $n=-4651,37+1,73 E B-42,83 \mathrm{FDN}$ & 37 & 0,71 \\
\hline Metodologia tradicional & $\mathrm{EMA}_{n}=-4942,00+1,94 \mathrm{~EB}-46,76 \mathrm{FDN}$ & 13 & 0,97 \\
\hline Todos os Sibbad & $\mathrm{EMA}_{n}=-4457,33+1,60 \mathrm{~EB}-40,21 \mathrm{FDN}$ & 21 & 0,88 \\
\hline \multicolumn{4}{|c|}{ Energia metabolizável verdadeira FDN } \\
\hline Todos os tratamentos & $\mathrm{EMV}=4157,18-47,04 \mathrm{FDN}$ & 38 & 0,75 \\
\hline Metodologia tradicional & $\mathrm{EMV}=4223,26-52,05 \mathrm{FDN}$ & 14 & 0,76 \\
\hline Todos os Sibbald & $\mathrm{EMV}=4113,13-40,91 \mathrm{FDN}$ & 22 & 0,76 \\
\hline \multicolumn{4}{|c|}{ Energia metabolizável verdadeira FDN +EB } \\
\hline Todos os tratamentos & $\mathrm{EMV}=-3506,78+1,66 \mathrm{~EB}-44,01 \mathrm{FDN}$ & 37 & 0,96 \\
\hline Metodologia tradicional & $\mathrm{EMV}=-4374,23+1,87 \mathrm{~EB}-48,65 \mathrm{FDN}$ & 13 & 0,97 \\
\hline Todos os Sibbald & $\mathrm{EMV}=-2928,47$ + 1,53EB - 40,91 FDN & 21 & 0,96 \\
\hline \multicolumn{4}{|c|}{ Energia metabolizável verdadeira corrigida FDN } \\
\hline Todos os tratamentos & $\mathrm{EMV}_{\mathrm{n}}=3931,02-46,14 \mathrm{FDN}$ & 38 & 0,73 \\
\hline Metodologia tradicional & $\mathrm{EMV}_{\mathrm{n}}=4028,55-50,26 \mathrm{FDN}$ & 14 & 0,73 \\
\hline Todos os Sibbald & $\mathrm{EMV}_{\mathrm{n}}=3865,99-43,40 \mathrm{FDN}$ & 22 & 0,74 \\
\hline \multicolumn{4}{|c|}{ Energia metabolizável verdadeira corrigida FDN +EB } \\
\hline Todos os tratamentos & $\mathrm{EMV}_{\mathrm{n}}=-4118,68+1,75 \mathrm{~EB}-42,95 \mathrm{FDN}$ & 37 & 0,96 \\
\hline Metodologia tradicional & $\mathrm{EMV}_{\mathrm{n}}=-4805,01+1,91 \mathrm{~EB}-46,75 \mathrm{FDN}$ & 13 & 0,97 \\
\hline Todos os Sibbald & $\mathrm{EMV}_{\mathrm{n}}=-3661,12+1,63 \mathrm{~EB}-40,42 \mathrm{FDN}$ & 21 & 0,97 \\
\hline
\end{tabular}

Para a EMA, quando se utilizaram os dados de todos os tratamentos, foi encontrado um $\mathrm{R}^{2}$ de 0,59 , idêntico ao $\mathrm{R}^{2}$ obtido para a EMA quando se utilizou somente a FB. O mesmo aconteceu quando se utilizaram dados dos tratamentos com metodologia tradicional e dados dos tratamentos de alimentação forçada. Para todas as outras variáveis $\left(E M A_{n}, E M V\right.$ e $\left.E M V_{n}\right)$, as equações obtidas com FDN foram muito parecidas àquelas obtidas com FB. Segundo Campbell et al. (1986), a FDN foi um preditor pior que 
a FB ou FDA. Como a análise de FDN é mais onerosa que a análise de FB, essa substituição não se justifica.

Carré et al. (1984) acreditam que a FDN subestima os carboidratos indigestíveis uma vez que essa medida não inclui as substâncias pépticas da parede celular, as quais não são digestíveis para aves, resultando em superestimativa da EM predita.

O mesmo pode ser dito com quanto à inclusão da EB ao modelo. Embora os $\mathrm{R}^{2}$ de todas as equações tenham se tornado maiores e, portanto, as equações mais precisas, a EB é uma análise cara, exige o uso de aparelhos de precisão, como bombas calorimétricas, e não é considerada uma análise de rotina. Os resultados obtidos pelas equações com FDN e EB são muito próximos daqueles obtidos com FB e EE, os últimos mais fáceis de serem analisados.

As equações de predição para $\mathrm{EMV}_{\mathrm{n}}$ deste trabalho, baseadas nas variáveis independentes FDN e EB, apresentaram maior precisão do que as encontradas por Zhang et al. (1994), os quais encontraram $\mathrm{R}^{2}$ de 0,62 e 0,64, ao utilizarem somente a FDN ou FDN mais EB, respectivamente.

Para simplificar, uma vez que o objetivo primário é agilizar o processo de obtenção da energia metabolizável, a recomendação é de que se utilize o mínimo de variáveis com o máximo de precisão. A utilização de muitas variáveis não promoveria aumento significativo da precisão e a utilização de uma só variável, no caso a FB, não aumentaria a segurança necessária para uma boa estimativa da EM.

Nesse caso, as equações eleitas para uma avaliação estão destacadas em negrito nas respectivas tabelas, a saber:

1- baseadas nos dados observados apenas nos tratamentos realizados a partir da metodologia tradicional:

$\mathrm{EMA}_{\mathrm{n}}=4337,0$ - 202,0 FB - 156,8 EE;

$\mathrm{EMV}=4542,6$ - 208,4 FB - 150,9 EE;

$\mathrm{EMV}_{\mathrm{n}}=$ 4381,4 - 201,9 FB - 155,7 EE;

2- baseadas nos dados observados apenas nos tratamentos de alimentação forçada com consumo igual:

$\mathrm{EMV}=4408,1$ - 178,2 FB - 129,7 EE;

$\mathrm{EMV}_{\mathrm{n}}=4198,6-180,9 \mathrm{FB}-138,4 \mathrm{EE}$.

As demais equações apresentadas, como por exemplo as multivariáveis, podem ser utilizadas, entretanto não melhoram a precisão dessas já destacadas.

Na Tab. 8 encontram-se os dados referentes às EMV dos alimentos observadas nos tratamentos 1, 2 e 3 obtidos por Borges et al. (2003) e estimadas pelas equações de predição de EMV a partir de análises químicas. As EMV estimadas foram iguais nos alimentos RR, GT e FTE quando comparadas às EMV observadas. Em TIM as equações apresentaram resultados inferiores aos observados nos três tratamentos. Para a FTC, o valor estimado de EMV pela equação obtida a partir da metodologia da alimentação forçada foi superior ao observado no tratamento com metodologia tradicional, entretanto foi semelhante ao resultado observado no tratamento 3. Para FTH, os valores estimados por todas as equações foram significativamente maiores do que os observados nos três tratamentos. Os valores estimados de EMV para o FTA pelas equações baseadas em todos os dados e pela equação baseada nos dados de alimentação forçada foram significativamente iguais, entretanto foi inferior quando estimada pela equação a partir dos dados experimentais obtidos pela metodologia tradicional.

Uma vez que os alimentos apresentaram diferenças entre tratamentos, principalmente no caso dos farelos, já se esperava que as equações também apresentassem algumas diferenças. No caso, somente o TIM apresentou valores observados de EMV maiores que aqueles estimados por todas as equações de EMV testadas. Uma hipótese aventada seria que a qualidade de nutrientes, ou ainda a interação entre os nutrientes desse alimento, permitiu digestibilidade maior in vivo que aquelas previstas pelas equações. 
Equações de regressão para estimar valores energéticos...

Tabela 8. Energia metabolizável verdadeira (EMV) dos alimentos observadas nos tratamentos 1 , 2 e 3 e estimadas pelas equações de predição de EMV a partir de análises químicas

\begin{tabular}{lcccc} 
& \multicolumn{3}{c}{ EMV estimada a partir dos dados experimentais com metodologia tradicional (FB e EE) } \\
\hline Alimento $^{1}$ & Trat. 1 & Trat. 2 & Trat. 3 & $4542,6-208,4$ FB-150,9 EE \\
\hline & & Determinada & Estimada \\
RR & $3417 \mathrm{a}$ & $3416 \mathrm{a}$ & $3341 \mathrm{a}$ & $3375^{\mathrm{a}}$ \\
TIM & $4273 \mathrm{a}$ & $4137 \mathrm{a}$ & $4224 \mathrm{a}$ & $3872 \mathrm{~b}$ \\
GT & $3094 \mathrm{a}$ & $2971 \mathrm{a}$ & $3068 \mathrm{a}$ & $2973 \mathrm{a}$ \\
FTC & $4113 \mathrm{~b}$ & $4066 \mathrm{~b}$ & $4156 \mathrm{~b}$ & $4389 \mathrm{a}$ \\
FTE & $3971 \mathrm{a}$ & $3962 \mathrm{a}$ & $3820 \mathrm{a}$ & $3954 \mathrm{a}$ \\
FTH & $1767 \mathrm{c}$ & $1737 \mathrm{c}$ & $1927 \mathrm{~b}$ & $2163 \mathrm{a}$ \\
FTA & $2002 \mathrm{a}$ & $2120 \mathrm{a}$ & $1819 \mathrm{~b}$ \\
FTG & $2107 \mathrm{c}$ & $2250 \mathrm{ab}$ & $2084 \mathrm{a}$ & $2182 \mathrm{~b}$ \\
\hline & EMV estimada a partir dos dados experimentais com a metodologia de alimentação forçada (FB e EE) \\
\hline & Trat. 1 & Trat. 2 & Trat. 3 & $4408,1-178,2$ FB-129,7 EE \\
\hline & & Determinada & $3341 \mathrm{a}$ & Estimada \\
RR & $3417 \mathrm{a}$ & $3416 \mathrm{a}$ & $3408 \mathrm{a}$ \\
TIM & $4273 \mathrm{a}$ & $4137 \mathrm{a}$ & $3824 \mathrm{a}$ & $383 \mathrm{~b}$ \\
GT & $3094 \mathrm{a}$ & $2971 \mathrm{a}$ & $3068 \mathrm{a}$ & $3059 \mathrm{a}$ \\
FTC & $4113 \mathrm{~b}$ & $4156 \mathrm{ab}$ & $4276 \mathrm{a}$ \\
FTE & $3971 \mathrm{a}$ & $4066 \mathrm{~b}$ & $3820 \mathrm{a}$ & $3902 \mathrm{a}$ \\
FTH & $1767 \mathrm{c}$ & $3962 \mathrm{a}$ & $1927 \mathrm{~b}$ & $2370 \mathrm{a}$ \\
FTA & $2002 \mathrm{~b}$ & $1737 \mathrm{c}$ & $2084 \mathrm{~b}$ & $2077 \mathrm{~b}$ \\
FTG & $2107 \mathrm{c}$ & $2120 \mathrm{a}$ & $2329 \mathrm{ab}$ & $2387 \mathrm{a}$
\end{tabular}

Valores na mesma linha seguidos de letras distintas diferem entre si pelo teste $\mathrm{SNK}(\mathrm{P}<0,05)$. FB= fibra bruta, $\mathrm{EE}=$ estrato etéreo. 1: RR= ração-referência, TIM= trigo integral moído, GT= germe de trigo, FTC= farinha de trigo clara, FTE= farinha de trigo escura, FTH= farelo de trigo para uso humano, FTA= farelo de trigo para uso animal, FTG= farelo de trigo grosso.

$\mathrm{Na}$ Tab. 9 encontram-se os dados referentes às $\mathrm{EMA}_{\mathrm{n}}$ e $\mathrm{EMV}_{\mathrm{n}}$ dos alimentos observadas nos tratamentos 1 , 2 e 3 por Borges et al. (2003) e estimadas pelas equações de predição de $E M A_{n}$ e $\operatorname{EMV}_{n}$ a partir de análises químicas. Os valores de predição de $\mathrm{EMA}_{\mathrm{n}}$, obtidos pela equação a partir da metodologia tradicional, foram diferentes de todos os valores do tratamento 3, o que já era esperado, uma vez que os tratamentos de alimentação forçada sofrem grande influência das perdas metabólica e endógena, sendo os valores de $\mathrm{EMA}_{\mathrm{n}}$ obtidos a partir dessa última metodologia subestimados. O TIM apresentou valores de $\mathrm{EMA}_{n}$ observados nos tratamentos com metodologia tradicional maiores que o estimado. Os valores observados na RR, GT, FTC e FTE nos tratamentos 1 e 2 foram semelhantes aos estimados. O valor estimado do FTH superestimou os valores observados nos tratamentos 1 e 2, enquanto que valores estimados para FTA e FTG foram semelhantes àqueles encontrados no tratamento com pintos, mas inferiores aos observados no tratamento com frangos.

Os dados estimados para a $\mathrm{EMV}_{\mathrm{n}}$ por todas as equações para o TIM foram menores que aqueles observados em todos os tratamentos. Para RR, GT, FTE e FTG, os valores estimados obtidos de todas as equações a partir de características químicas (Tab. 9) foram semelhantes aos observados em todos os tratamentos.

A FTC apresentou resultados estimados de $\mathrm{EMV}_{\mathrm{n}}$ iguais $(\mathrm{P}>0,05)$ aos observados nos tratamentos, quando as equações utilizadas foram baseadas em todos os dados experimentais e nos dados do tratamento de alimentação forçada, entretanto apresentou valores estimados mais altos $(\mathrm{P}<0,05)$ quando estimada pela equação baseada nos dados dos tratamentos obtidos a partir da metodologia tradicional.

O FTH apresentou valores de $\mathrm{EMV}_{\mathrm{n}}$ estimados mais altos que os observados em todas as equações e o FTA apresentou resultados estimados semelhantes aos observados somente no tratamento 1, com pintos. Os valores observados nos outros tratamentos para o FTA foram estatisticamente maiores que o estimado. 


\section{Borges et al.}

Tabela 9. Energia metabolizável aparente corrigida $\left(\mathrm{EMA}_{\mathrm{n}}\right)$ e energia metabolizável verdadeira corrigida $\left(\mathrm{EMV}_{\mathrm{n}}\right)$ dos alimentos observadas nos tratamentos 1 , 2 e 3 e estimadas pelas equações de predição de $\mathrm{EMA}_{\mathrm{n}}$ e $\mathrm{EMV}_{\mathrm{n}}$ a partir de análises químicas

\begin{tabular}{|c|c|c|c|c|}
\hline \multicolumn{5}{|c|}{ EMA $_{n}$ estimada a partir dos dados experimentais com metodologia tradicional (FB e EE) } \\
\hline & Trat. 1 & Trat. 2 & Trat. 3 & 4337,0-202,0 FB-156,8 EE \\
\hline & \multicolumn{3}{|c|}{ Determinada } & Estimada \\
\hline $\mathrm{RR}$ & $3121 \mathrm{a}$ & $3200 \mathrm{a}$ & 2084b & 3169a \\
\hline TIM & 4059a & $4001 \mathrm{a}$ & 3003c & $3667 b$ \\
\hline GT & $2758 \mathrm{a}$ & $2785 a$ & $1846 b$ & $2706 a$ \\
\hline FTC & 3898a & $3881 a$ & 2838b & $4177 a$ \\
\hline FTE & 3706a & $3754 a$ & $2576 b$ & $3732 a$ \\
\hline FTH & $1557 b$ & $1614 b$ & $860 c$ & $1977 a$ \\
\hline FTA & 1789b & $1938 a$ & $982 c$ & $1655 b$ \\
\hline \multirow[t]{4}{*}{ FTG } & $1911 b$ & $2105 a$ & $1148 \mathrm{c}$ & $2007 a$ \\
\hline & \multicolumn{4}{|c|}{ EMV $_{\mathrm{n}}$ estimada a partir dos dados experimentais com metodologia tradicional (FB e EE) } \\
\hline & Trat. 1 & Trat. 2 & Trat. 3 & 4381,4-201,9 FB-155,7 EE \\
\hline & \multicolumn{3}{|c|}{ Determinada } & Estimada \\
\hline $\mathrm{RR}$ & $3187 \mathrm{ab}$ & $3231 \mathrm{a}$ & $3067 \mathrm{~b}$ & $3217 \mathrm{a}$ \\
\hline TIM & 4111a & 4024a & 4010a & $3714 \mathrm{~b}$ \\
\hline GT & 2846a & $2809 a$ & 2828a & $2762 \mathrm{a}$ \\
\hline FTC & 3966b & $3922 b$ & 3839b & 4223a \\
\hline FTE & 3770a & $3782 a$ & 3566a & $3780 a$ \\
\hline FTH & $1639 c$ & $1646 c$ & 1838b & $2028 a$ \\
\hline FTA & 1860a & $2006 a$ & 1958a & $1704 \mathrm{~b}$ \\
\hline \multirow[t]{4}{*}{ FTG } & 1990a & $2132 a$ & 2141a & $2056 a$ \\
\hline & \multicolumn{4}{|c|}{ EMV $_{\mathrm{n}}$ estimada a partir dos dados experimentais com a metodologia de alimentação forçada (FB e EE) } \\
\hline & Trat. 1 & Trat. 2 & Trat. 3 & 4198,6-180,9 FB-138,4 EE \\
\hline & \multicolumn{3}{|c|}{ Determinada } & Estimada \\
\hline $\mathrm{RR}$ & 3187ab & $3231 \mathrm{a}$ & $3067 \mathrm{~b}$ & 3159a \\
\hline TIM & 4111a & 4024a & 4010a & $3603 b$ \\
\hline GT & 2846a & $2809 a$ & $2828 a$ & $2759 a$ \\
\hline FTC & 3966a & $3922 a$ & 3839a & $4057 a$ \\
\hline FTE & $3770 a$ & $3782 a$ & 3566a & 3663a \\
\hline FTH & 1639c & $1646 c$ & 1838b & $2095 a$ \\
\hline FTA & $1860 \mathrm{ab}$ & $2006 a$ & 1958a & $1805 b$ \\
\hline FTG & 1990a & $2132 a$ & $2141 a$ & $2120 \mathrm{a}$ \\
\hline
\end{tabular}

Valores na mesma linha seguidos de letras distintas diferem entre si pelo teste $\mathrm{SNK}(\mathrm{P}<0,05) . \mathrm{FB}=$ fibra bruta, $\mathrm{EE}=$ estrato etéreo. 1: RR= ração-referência, TIM= trigo integral moído, GT= germe de trigo, FTC= farinha de trigo clara, FTE= farinha de trigo escura, FTH= farelo de trigo para uso humano, FTA= farelo de trigo para uso animal, FTG= farelo de trigo grosso.

Pelos resultados estimados pelas equações a partir das análises químicas pode-se notar que todas mostraram tendência de apresentar valores observados semelhantes aos estimados tanto para EMV como para $\mathrm{EMV}_{\mathrm{n}}$.

Assim como a metodologia experimental pode afetar os valores obtidos de energia metabolizável, pode também afetar os resultados obtidos a partir das equações de predição, uma vez que são desenvolvidas a partir dos dados obtidos em ensaios experimentais. Daí a necessidade de se encontrar uma metodologia que reflita, com a maior fidelidade possível, a energia metabolizável para aves. A outra questão seria obter, em futuras pesquisas, equações para diferentes estádios fisiológicos, uma vez que a energia metabolizável pode ser distinta para um mesmo alimento em pintos e aves adultas, principalmente em alimentos ricos em fibra.

\section{CONCLUSÕES}

Os resultados obtidos neste trabalho conduziram às seguintes conclusões: 1- a FB foi a variável que melhor se relacionou com os valores de energia metabolizável, entretanto esse valor isolado não é 
suficiente para uma boa estimativa dos valores energéticos; 2- a precisão das equações aumentou quando foram adicionados os valores de EE e PB; 3- as equações estimadas a partir dos valores de todos os tratamentos resultaram em equações menos precisas para EMA devido ao efeito dos valores obtidos nos tratamentos de alimentação forçada, fortemente afetados pelo nível de consumo; 4- a utilização somente de dados dos tratamentos com metodologia tradicional ou de alimentação forçada separadamente aumentou a precisão das equações; 5- para $\mathrm{EMV}$ e $\mathrm{EMV}_{\mathrm{n}}$ as equações lineares múltiplas, utilizando-se da FB, EE e PB, apresentaram boa precisão, independente da utilização de todos os dados experimentais ou seu agrupamento por metodologia; 6- FDN não mostrou ser melhor preditor de EM que FB; 7- os valores de EM estimados podem se apresentar diferentes dos observados em alguns alimentos em função das diferenças encontradas nos valores observados nos alimentos entre os tratamentos.

\section{REFERÊNCIAS BIBLIOGRÁFICAS}

ALBINO, L.F.T. Determinação de valores de energia metabolizável e triptofano de alguns alimentos para aves em diferentes idades. 1980. 55p. Dissertação (Mestrado). Universidade Federal de Viçosa, Viçosa, MG.

BORGES, F.M.O.; ROSTAGNO, H.S.; SAAD, C.E.P. et al. Comparação de métodos de avaliação dos valores energéticos do grão de trigo e seus subprodutos para frangos de corte. Arq. Bras. Med. Vet. Zootec., v.55, p.710-721, 2003.

CAMPBELL, G.L.; SALMON, R.E.;, CLASSEN, H.L. Prediction of metabolizable energy of broiler diets from chemical analysis. Poult. Sci., v.65, p.2126-2134, 1986.

CARRÉ, B.; PREVOTEL, B.; LECLERCQ, B. Cell wall content as a predictor of metabolisable energy value of poultry feedingstuffs. Br. Poult. Sci., 25, p.561-572, 1984.

NUTRIENT requirements of poultry. 8.ed. Washington: National Academy Press, 1994. 155p.

ROSTAGNO, H.S. Valores de alimentos e de exigências nutricionais utilizados na formulação de rações para aves. In: REUNIÃO ANUAL DA SOCIEDADE BRASILEIRA DE ZOOTECNIA, Campinas, 1990. Anais... Viçosa: SBZ, 1990. p.11-30.

SAKAMURA, N.K.; SILVA, R. Conceitos inovadores aplicáveis à nutrição de não ruminantes. Cad. Téc. Esc. Vet. UFMG, v.22, p.125-146, 1998.

SIBBALD, I.R.; PRICE, K. True and apparent metabolizable energy values for poultry of canadian wheats and oats measured by bioassay and predicted from physical and chemical data. Can. J. Anim. Sci., v.57, p.365-374, 1977.

SIBBALD, I.R.; PRICE, K. True metabolizable energy values for poultry of Canadian barleys measured by bioassay and predicted from physical and chemical data. Can. J. Anim. Sci., v.56, p.775-782, 1976.

ZHANG, W.J.; CAMPBELL, L.D.; STOTHERS, S.C. An investigation of the feasibility of predicting nitrogen-corrected true metabolizable energy (TMEn) content in barley from chemical composition and physical characteristics. Can. J. Anim. Sci., v.74, p.355-360, 1994. 